\title{
EVALUATION OF STRAIN DEVELOPED AROUND SHORT DENTAL IMPLANTS USING TWO DIFFERENT RESTORATIVE MATERIALS (IN VITROSTUDY)
}

\author{
Eman A Ibrahim ${ }^{1 *} B D S$, Ahlam Elsharkawy² $P H D$, Mohamed S Nassif ${ }^{3}$ PHD, Nermeen A \\ $\operatorname{Rady}^{4} P H D$
}

ABSTRACT

INTRODUCTION: The use of short implants has been introduced as an alternative treatment for posterior regions, however, it leads to serious prosthetic complications. Using CAD/CAM, materials like zirconia and Bio-HPP can be used to fabricate implant supported restorations. OBJECTIVES: This study aimed to evaluate and compare the strains developed around short and standard implant length using two different crown materials.

MATERIALS AND METHODS: Polyurethane blocks $(n=20)$ were used as alternative materials for human cancellous bone. Blocks were divided into two groups, group A received ten standard length implants $12 \mathrm{~mm}$, and group B received ten short implants $7 \mathrm{~mm}$. Each group was equally subdivided into two subgroups, according to crown material (BioHPP and zirconia). Universal testing machine was used to apply a load of $100 \mathrm{~N}$ axially and obliquely at $45^{\circ}$ on the restorations. Microstrains were measured using strain meter.

RESULTS: The difference in microstrain values between BioHPP and zirconia was statistically insignificant for both group A and group B. Comparing between group A and group B having the same restorative materials, it was found that, the difference was statistically significant for zirconia in axial loading only. A significant difference was observed between oblique and axial loads in standard implant length for both BioHPP and zirconia restorations, and for zirconia in short implants as well (p value $=0.043$ ), while the difference was insignificant for BioHPP in short implants.

CONCLUSIONS: Short implants are comparable treatment modality to standard implant lengths for single tooth restoration. Oblique forces produce more stresses than vertical forces. According to the average of loads, there is no significant difference between BioHPP and zirconia for both short and standard implant length. However, it is advisable not to use zirconia restorations with short implants.

KEYWORDS: Short implants, Crown/implant ratio, CAD/CAM, Zirconia, High Performance Polymer, Strain gauge.

1. Eman Assem Ibrahim, instructor at prosthodontic department, Faculty of Dentistry, Alexandria University, Alexandria, Egypt.

2. Professor Dr. Ahlam El Sharkawy, Professor at Prosthodontics department, Faculty of Dentistry, Alexandria University, Alexandria, Egypt.

3. Professor Dr. Mohamed Salah Nassif, Assistant Professor at Biomaterials department, Faculty of dentistry, University of Ain Shams, Cairo, Egypt.

4. Dr. Nermeen Abd El Salam Rady, Lecturer at prosthodontics department, Faculty of dentistry, Alexandria University, Alexandria, Egypt.

*Corresponding author:

E-mail: emanassemibrahim@gmail.com

\section{INTRODUCTION}

During the last decade, implantology has become an essential part of dental restorations, helping dentists to improve the quality of life of large populations. The use of dental implants is often the treatment of choice to replace missing teeth in partially edentulous patients.

It is also well known that, occlusal stresses should be kept within physiological limits when planning implants supported restorations as it might affect the outcome of implant restorations. Load transfer is influenced by bone quality, implant length and design, the amount of occlusal load as well as the abutments and prosthesis design and material (1).

With the loss of teeth, the alveolar ridge undergoes a continuous and irreversible process of bone resorption in height and thickness. Usually, the posterior bone resorption leads to reduced bone height, therefore the placement of implants in these regions becomes challenging (2).

Generally, the placement of a standard length implant without bone augmentation requires a minimum residual bone height of $8 \mathrm{~mm}$. Thus, a successful implant treatment in the mandible can be limited in posterior regions due to insufficient bone height. Any attempt to place standard length implant in such regions, will increases the risk of damaging the inferior alveolar nerve. To avoid this, several methods have been developed for the rehabilitation of atrophic jaws with the installation of dental implants, such as; placement of tilted implants (3), distraction osteogenesis (4), bone grafts, guided bone regeneration (5), and lateralization of the inferior alveolar nerve (6).

Recently, short implants concept has emerged as a successful alternative treatment option in sites thought unfavorable for implant success, such as those associated with bone resorption. Since surgeries include high morbidity, require long clinical procedure and have higher costs.

It is clear that, short implants are less complex and less invasive treatment than placement of longer implants in clinical sites where adjunctive procedures would be required. They are also less traumatic. This type of implants provides faster and less expensive treatment with less morbidity. In fact, the stability of short implant proved enough ability to support the occlusal force (7).

There is no consensus about the definition of short implants. They may be considered to be $<10 \mathrm{~mm}$ (8), or $\leq 8$ $\mathrm{mm}$ (9). Current clinical tendencies consider implants with 
$7 \mathrm{~mm}$ length or less as short or extra-short implants (10). On the other hand, since implants of $10 \mathrm{~mm}$ length have proven predictable success, they are commonly considered to be standard length, and so any implant below $10 \mathrm{~mm}$ is referred as a short implant (11).

The concept that crown/implant (C/I) ratios should not exceed the guidelines established for natural teeth, has not been documented. Guidelines for unfavorable C/I ratios do not exist in the dental literature. A design factor that is closely related to the $\mathrm{C} / \mathrm{I}$ ratio is implant length (12).

The measurements of the $\mathrm{C} / \mathrm{I}$ ratio were approached differently; the anatomical $\mathrm{C} / \mathrm{I}$ ratio, which considered the fulcrum established at the interface between the implant shoulder and the crown-abutment complex (13), and the clinical $\mathrm{C} / \mathrm{I}$ ratio, where the fulcrum is positioned at the most coronal bone-implant contact. The anatomical C/I ratio offers a more favorable biomechanical scenario, as the lever arm is shorter than in the clinical C/I ratio. However, the clinical C/I ratio offers a more realistic clinical scenario, as the connection of the implant restoration at the implant shoulder is more rigid than at the implant-bone interface due to bone viscoelasticity (14).

In the literature, there is a considerable debate on the influence of $\mathrm{C} / \mathrm{I}$ ratio on short implant success. Based on the fact that, each crown has to be constructed to reach the occlusal plane, the use of short implants definitely causes a higher C/I ratio, which might increase the risk of biomechanical complications. The negative influence of increased C/I ratio was reported (15). However, other studies $(16,17)$ proved that, there is no influence on crestal bone loss, and that, $\mathrm{C} / \mathrm{I}$ ratio is not a suitable predictor of implant survival.

Computer-aided technology can use computer skills to design and manufacture products. It either involve additive or subtractive manufacturing. In prosthodontics, the subtractive procedure, is widely used. Computer aided designing/ computer aided manufacturing (CAD/CAM) is well known for its accuracy and reproducibility, it is fast and can avoid the errors that occur during casting.

Advanced systems have enabled the fabrication of restorations from high performance polymers. Poly-etherether-ketone (PEEK) is a synthetic thermoplastic polymer that has been successfully used in the medical field. The material exhibits high mechanical performance, and has been recently introduced in dentistry. A newly modified PEEK material containing 20\% ceramic fillers is BioHPP, which presents high biocompatibility, good mechanical properties, high temperature resistance, and chemical stability. It is fabricated either with CAD/CAM or with compression molding (18).

It is as elastic as bone and can reduce stresses transferred to the abutment teeth and peripheral bone. Furthermore, the white color of BioHPP frameworks provides a different esthetic approach (19).

Additionally, another restorative material that has been repeatedly used in dental restorations is zirconia. Clearly, zirconia materials have the advantage of being aesthetic, highly biocompatible and a thermal insulator. Furthermore, zirconia has a high flexural strength and fracture toughness, which are significantly higher than any other ceramic system. In fact, zirconia frameworks present low bacterial surface adhesion (20). Recently, it can be fabricated with a minimal occlusal thickness of $0.5 \mathrm{~mm}$ that can withstand a bite force in the molar region.
Strain gauges are the most widely used in experimental mechanics to evaluate strain at a point on engineering structures. The accuracy of the strain gauges is maintained without failures due to loss of retention of the gauges. The small size of strain gauges and their minimal interference during function make them ideal for measuring deformation under clinical conditions (21).

\section{MATERIALS AND METHODS}

Solid rigid polyurethane foam blocks (Aptic Medical, Washington, USA); 20 pcf (pound per cubic foot) are synthetic blocks that are produced as an alternative test medium to human cancellous bone $(22,23)$. The uniformity and consistent properties of rigid polyurethane foam for comparative testing blocks are $12.5 \mathrm{~cm} \times 5 \mathrm{~cm} \times 5.5 \mathrm{~cm}$. Four blocks were sawed using electric saw. Twenty blocks were obtained, $2 \mathrm{~cm}$ length, $5 \mathrm{~cm}$ width and $4.5 \mathrm{~cm}$ height dimensions.

A model of bounded saddle was created, including the crown portion of left maxillary canine and left maxillary second premolar with missing left maxillary first premolar. The crowns were connected with a bar from the palatal surface. The edentulous space between the two crowns was adjusted at $8.5 \mathrm{~mm}$ using a caliber to match the diameter of the implant that is to be placed, and preserve $1.5 \mathrm{~mm}$ away from each adjacent tooth. The $8.5 \mathrm{~mm}$ edentulous space was standardized in all the models.

Then, twenty final virtual models were printed out using special 3D dental printer with specific material (Planmeca Creo, dental model, Netherlands). The printed models were then finished with low speed hand piece to remove excess material. The models were placed in post curing light pulsing unit, and were fixed on the twenty polyurethane test blocks using cyanoacrylate adhesive.

The implant position was virtually planned using implant studio software. The implant vertical axis was made parallel to the long axis of the adjacent teeth, and in the middle of the tooth occlusal plane.

The surgical guide was directly modelled on the final virtual model of the teeth. A uniform layer of material was added to the reference surface of the virtual model using implant studio software. The surgical guide design was printed. One surgical guide was fabricated from specific material (next dent surgical guide resin, Netherlands). Drilling sleeve was then inserted and glued in the guide, which represented the actual guiding system.

Blocks were randomly divided into two main groups, according to the implant (Dentis S-Clean, korea) length. Each group was divided into two subgroups according to the crown material.

Group A (Control Group): Ten $5.5 \mathrm{~mm}$ dental implants diameter, and $12 \mathrm{~mm}$ length.

Subgroup (1): Five implants were restored with Bio-HPP (bredent, Germany) crowns.

Subgroup (2): Five implants were restored with zirconia (Kuraray Noritake, Tokyo, Japan) crowns.

Group B (Study Group): Ten $5.5 \mathrm{~mm}$ dental implants diameter, and $7 \mathrm{~mm}$ length.

Subgroup (1): Five implants were restored with Bio-HPP crowns.

Subgroup (2): Five implants were restored with zirconia crowns. 
In Group $\mathrm{A}$, the surgical guide was placed on the polyurethane block and through the metal sleeve; a pilot drill of $2 \mathrm{~mm}$ diameter was inserted to drill the pilot hole. Then, a number of drills $(2.3 \mathrm{~mm}, 3 \mathrm{~mm}, 3.4 \mathrm{~mm}, 3.8 \mathrm{~mm}$, $4 \mathrm{~mm}$, and $4.2 \mathrm{~mm}$ ) was used sequentially to increase the diameter of the hole in order to match the diameter of the implant (24). The length of the drills was $16 \mathrm{~mm}$, so a drill stop of $2 \mathrm{~mm}$ was added to the drills to match the $12 \mathrm{~mm}$ standard implant length. The final depth of the hole was confirmed using depth gauge.

Ten implants of $5.5 \mathrm{~mm}$ diameter, $12 \mathrm{~mm}$ length were screwed in ten polyurethane blocks with implant driver till the implant became partially stable. The final insertion was then achieved by using torque wrench of $35 \mathrm{Ncm}$ as recommended by the manufacturer.

The same was applied for short implants in Group B except that, the length of the drills was $10 \mathrm{~mm}$ and a drill stop of $1 \mathrm{~mm}$ was added to the drills to match the $7 \mathrm{~mm}$ short implant length. The final depth of the hole was confirmed using depth gauge.

Titanium straight abutments (Dentis S-Clean, South Korea) of $4.5 \mathrm{~mm}$ diameter, collar $1.5 \mathrm{~mm}$, and height $7 \mathrm{~mm}$ were screwed using abutment screw driver and torque wrench with $30 \mathrm{Ncm}$ torque as recommended by the manufacturer.

Bio-HPP and Zirconia crowns of the maxillary first premolar were fabricated using CAD/CAM system to standardize their dimensions; titanium abutments were sprayed with special spray (Dentech lab supplies LTD, Auckland, USA) to be captured and scanned with an extraoral scanner (inEos X5, Dentsply Sirona, Philadelphia, USA). Virtual model for the crowns were created on the software. ST files of the virtual designs of the crowns were transferred to milling CAD/CAM machine (Dentsply Sirona, Philadelphia, USA) to start the milling process of BioHPP and zirconia discs. The crowns were adapted and prepared for cementation.

The crowns were checked for their passive fit on the abutments. Abutments were sandblasted using $110 \mu \mathrm{m}$ $\mathrm{Al}_{2} \mathrm{O}_{3}$ powder (PROTECHNO, Spain) inside sandblasting chamber as recommended to be prepared for cementation followed by a layer of zirconia primer (Z-prime plus, USA). The adhesive surfaces of the restoration made from BioHPP were pretreated by blasting with aluminum oxide $(110 \mu \mathrm{m}$ $\mathrm{Al} 2 \mathrm{O} 3$ ) at 2 to 3 bar blasting pressure. Moistening then took place with light-hardened PMMA and Composite Primer (Visio. Link, Germany) and subsequent polymerization (e.g. 90 seconds) in accordance with the "Visio. Link" processing instructions, while the adhesive surface of zirconia crowns was pretreated with aluminum oxide (110 $\mu \mathrm{m} \mathrm{Al}_{2} \mathrm{O}_{3}$ ) then a layer of zirconia primer (Z-prime plus, Bisco Dental, Illinois, USA) was placed. Resin cement was then placed inside the restorations and light-cured for cementation.

Two channels were drilled on the facial and palatal surface of all the polyurethane blocks. The channels were made opposite to the implant site such that; their depth was $2 \mathrm{~mm}$ away from the implant.

Two linear strain gauges (Kyowa Electronic Instruments, Japan) were bonded buccal and lingual to the polyurethane test blocks adjacent to the implant site using a cyanoacrylate adhesive to measure the strain around the implant.
The terminals of the two strain gauge wires were connected to a circuit multichannel strain meter (Kyowa sensor interface PCD-300A, Japan) to record the developed strain. The strain meter was connected to a computer screen. A universal testing device (Lloyd instruments LR 5K, UK) was used to apply static load on the first premolar crown (25). A single point of a $100 \mathrm{~N}$ vertical static load was applied at a constant rate. The point of axial load application was selected at the site of:

1. The central fossa of the crown.

2. The buccolingual midpoint of the mesial marginal ridge. 3. The buccolingual midpoint of the distal marginal ridge.

A self-developed jig was designed with an adjustable rotational screwing device so that a $45^{\circ}$ lateral force could be applied in the experiments (26). Each loading procedure involved applying a force of $100 \mathrm{~N}$ using a universal testing machine. The strain meter measured the strain developed and the results were recorded on the computer.

\section{Statistical Analysis}

Data were fed to the computer and analyzed using IBM SPSS software package version 20.0 (Armonk, NY: IBM Corp). Quantitative data were analyzed using minimum and maximum, mean, standard deviation and median of microstrain. To compare between groups and subgroups, the nonparametric Kruskal-Wallis test was used. If significant differences were detected, the Mann-Whitney U test was used for pairwise comparisons (post hoc test). Values were considered significant when $p$ value is $\leq 0.05$.

\section{RESULTS}

This study was conducted to evaluate and compare between the strain developed around standard length implants (length $12 \mathrm{~mm}$, diameter $5.5 \mathrm{~mm}$ ) and short dental implants (length $7 \mathrm{~mm}$, diameter $5.5 \mathrm{~mm}$ ) using two different crown materials which were fabricated using CAD/CAM system when loads are applied at different locations.

Figure 1 shows the summary of the mean value of the average microstrain that was developed buccally and palatally at different load application points (axial load on the central fossa, axial load on the mesial marginal ridge, axial load on the distal marginal ridge and oblique load $45^{\circ}$ on the central fossa).

Comparing between the two subgroups in group A and group B, when vertical load was applied on central fossa, the difference of microstrain values between the two subgroups was statistically insignificant with p-value equals to 0.769 and 0.688 , respectively.

In addition, comparing between group A and group B, according to BioHPP restorations, it was found that, the difference in the microstrains developed around the implants was insignificant ( $\mathrm{p}$-value=0.051). Adversely, the difference in microstrain values was statistically significant for zirconia restorations, with p-value equals to 0.040 .

For mesial load application, when comparing between the two subgroups of group $A$, it was found that, the difference was insignificant ( $p$-value $=0.708$ ). As for group $\mathrm{B}$, the difference was also found to be statistically insignificant ( $\mathrm{p}$-value $=0.630)$.

For the BioHPP restorative material between group A and $\mathrm{B}$, no significant difference was found between the two groups $(p$-value $=0.087)$. While for zirconia restorations, the difference of the microstains developed between the two 
groups was statistically significant with p-value equals to 0.010 .

Comparing between subgroups of group A, when distal load was applied, it was found that, the difference was insignificant ( $\mathrm{p}$ value $=0.487$ ). Besides, for group $\mathrm{B}$, the $\mathrm{p}$ value was found to be statistically insignificant ( $\mathrm{p}$ value $=0.708$ ).

According to BioHPP between group A and group B, there was no significant difference between the two groups ( $p$ value $=0.134$ ). On the other hand, comparing between the two groups, when using zirconia, the difference was found to be statistically significant ( $p$ value $=0.010$ ).

Considering oblique load that was applied at $45^{\circ}$ on the central fossa, when comparing between subgroups of group $A$, it was found that, the difference in microstrain values was found to be insignificant. (p-value $=0.917$ ). The same was applied for group B, and the difference was also found to be insignificant with p-value equals to 0.753 .

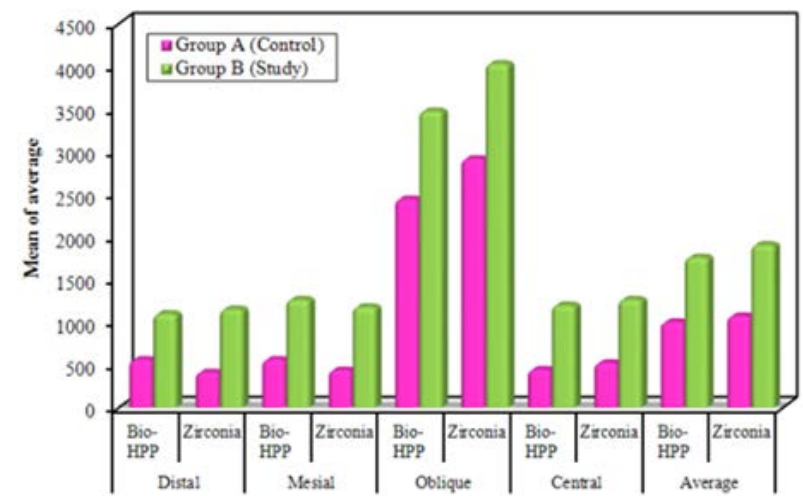

Figure (1): Summary of the mean value of the average microstrain that was developed buccally and palatally at different load application points.

Upon comparing between group $\mathrm{A}$ and group $\mathrm{B}$, according to BioHPP and zirconia restorations, the difference was found to be statistically not significant for both materials.

According to the average of all types of load application, when comparing between the subgroups, it was found that, the difference was statistically insignificant for both group A and group B. (Table 1)

Table (1): Summary of the mean value of the average microstrain that was developed buccally and palatally at different load application points

\begin{tabular}{|r|c|c|c|c||}
\hline \multirow{2}{*}{ Average } & \multicolumn{2}{|c|}{ Group A (Control) } & \multicolumn{2}{c||}{ Group B (Study) } \\
\cline { 2 - 5 } & Bio-HPP (n = 5) & $\begin{array}{c}\text { Zirconia (n } \\
=5)\end{array}$ & $\begin{array}{c}\text { Bio-HPP (n } \\
=5)\end{array}$ & $\begin{array}{c}\text { Zirconia (n } \\
\text { 5) }\end{array}$ \\
\hline Distal & $562.5^{\mathrm{ab}}$ & $415.0^{\mathrm{b}}$ & $1101.0^{\mathrm{a}}$ & $1155.0^{\mathrm{a}}$ \\
\hline Mesial & $563.0^{\mathrm{bc}}$ & $440.0^{\mathrm{c}}$ & $1266.0^{\mathrm{ab}}$ & $1180.5^{\mathrm{a}}$ \\
\hline Oblique & $2445.5^{\mathrm{a}}$ & $2918.5^{\mathrm{a}}$ & $3477.5^{\mathrm{a}}$ & $4032.5^{\mathrm{a}}$ \\
\hline Central & $446.5^{\mathrm{b}}$ & $525.0^{\mathrm{b}}$ & $1201.5^{\mathrm{ab}}$ & $1268.0^{\mathrm{a}}$ \\
\hline Average & $1004.4^{\mathrm{a}}$ & $1074.6^{\mathrm{a}}$ & $1761.5^{\mathrm{a}}$ & $1909.0^{\mathrm{a}}$ \\
\hline
\end{tabular}

Means with Common letters are not significant (i.e. Means with Different letters are significant)

In addition, comparing between group $\mathrm{A}$ and group $\mathrm{B}$ having same restorative material, it was found that, the differences were statistically not significant for both
BioHPP and zirconia with p-values equals to 0.117 and 0.056 , respectively.

Table 2 shows the minimum and maximum, mean, standard deviation and median of the microstrain values developed on comparing between the average of central and oblique loads in short and standard implant length.

When comparing between the mean values of microstrains of the average of central and oblique loads in group A, according to BioHPP and zirconia restorations, it was found that, the difference between the two types of loads was statistically significant with p-value equals 0.043 .

As for Group B, the same comparison was made, according to BioHPP, and the difference was found to be statistically not significant ( $\mathrm{p}$-value $=0.080$ ). However, the difference was found to be statistically significant for zirconia restoration with p-value equals 0.043 . (Figure 2)

Table (2): Comparison between average of oblique and central

\begin{tabular}{|c|c|c|c|c|}
\hline & \multicolumn{2}{|c|}{ Oblique } & \multicolumn{2}{|c|}{ Central } \\
\hline & $\begin{array}{c}\text { Bio-HPP (n } \\
=5)\end{array}$ & $\begin{array}{c}\text { Zirconia } \\
(\mathrm{n}=5)\end{array}$ & $\begin{array}{c}\text { Bio-HPP (n } \\
=5)\end{array}$ & $\begin{array}{c}\text { Zirconia } \\
(n=5)\end{array}$ \\
\hline $\begin{array}{l}\text { Group A } \\
\text { (Control) }\end{array}$ & & & & \\
\hline $\begin{array}{l}\text { Median } \\
\text { (Min. - } \\
\text { Max.) }\end{array}$ & $\begin{array}{c}3377.5(522 . \\
5-3840.0)\end{array}$ & $\begin{array}{c}2127.5(194 \\
5-6262.5)\end{array}$ & $\begin{array}{c}515.0(130.0 \\
-745.0)\end{array}$ & $\begin{array}{c}537.5(12 \\
7.5- \\
1155.0)\end{array}$ \\
\hline $\begin{array}{l}\text { Mean } \pm \\
\text { SD. }\end{array}$ & $\begin{array}{c}2445.5 \pm 152 \\
0.1\end{array}$ & $\begin{array}{c}2918.5 \pm 18 \\
75.2\end{array}$ & $446.5 \pm 232.2$ & $\begin{array}{c}525.0 \pm 40 \\
1.4\end{array}$ \\
\hline${ }^{z_{p}}$ & & & $0.043^{*}$ & $0.043^{*}$ \\
\hline $\begin{array}{l}\text { Group B } \\
\text { (Study) }\end{array}$ & & & & \\
\hline $\begin{array}{l}\text { Median } \\
\text { (Min. - } \\
\text { Max.) }\end{array}$ & $\begin{array}{c}3765.0(582 \\
5-6760.0)\end{array}$ & $\begin{array}{c}3370.0(294 \\
7.5-6035)\end{array}$ & $\begin{array}{c}957.5(230.0 \\
-2167.5)\end{array}$ & $\begin{array}{c}1380.0(6 \\
95.0- \\
1680.0)\end{array}$ \\
\hline $\begin{array}{l}\text { Mean } \pm \\
\text { SD. }\end{array}$ & $\begin{array}{c}3477.5 \pm 227 \\
9.2\end{array}$ & $\begin{array}{c}4032.5 \pm 13 \\
68.6\end{array}$ & $\begin{array}{c}1201.5 \pm 750 \\
13\end{array}$ & $\begin{array}{c}1268.0 \pm 3 \\
83.4\end{array}$ \\
\hline${ }^{z_{p}}$ & & & 0.08 & $0.043^{*}$ \\
\hline
\end{tabular}

${ }_{\mathrm{z}} \mathrm{p}$ : $\mathrm{p}$ value for Wilcoxon signed ranks test for comparing between oblique and central

*: Statistically significant at $\mathrm{p} \leq 0.05$

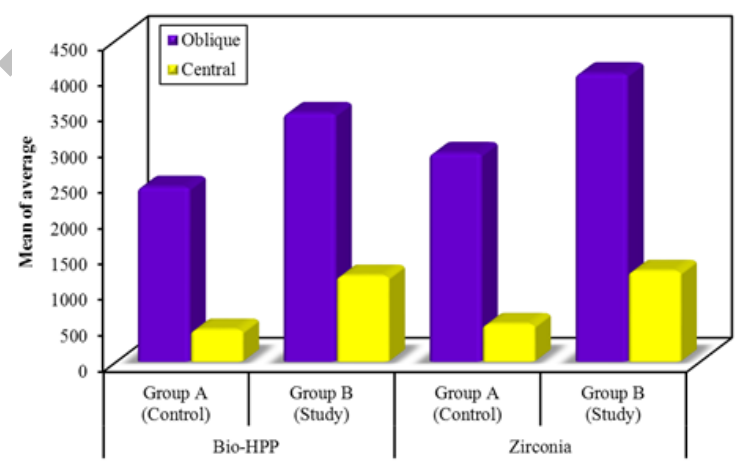

Figure (2): Comparison between average of oblique and central

\section{DISCUSSION}

Solid rigid polyurethane foam was selected for this study as an alternative test medium for human cancellous bone. It provides consistent mechanical properties in the range of human cancellous bone. The uniformity and consistent properties of rigid polyurethane foam make it an ideal material for comparative testing of bone screws (22).

Moreover, a CAD/CAM guide was designed to control the drill angulation, depth, and location of the implant. The guided surgery is well known for its high accuracy in implant placement. The surgical guide was made from rigid 
transparent material to be stabilized in its position during drilling and to allow easier observation of the blocks and drills (27).

The conventional drilling protocol was followed using sequential drills. The drilling protocol was customized by underpreparing the width of the implant site, according to the block density to obtain primary stability.

The development of CAD/CAM technology has been dramatic in achieving distinct criterion for successful dental restorations. In this manner, BioHPP and Zirconia have been selected as restorative materials for both short and standard implant lengths. BioHPP is a synthetic thermoplastic polymer that exhibits high mechanical performance. The compatibility between the elastic modulus of the BioHPP and bone may reduce the stress effects on peripheral bone (28).

Furthermore, monolithic zirconia, has many advantages including; significant biocompatibility, superior mechanical properties as well as good esthetics. Zirconium is used mainly in high stress areas such as the posterior region (29).

In this study, the strain gauge analysis was used to evaluate strain around abutment teeth as it provides quantitative analysis of the strain. Using strain gauges for measuring strains and deformation due to their small size, linearity, and minimal interference during testing.

The loads applied during the experiment were below maximum masticatory forces recorded for humans, as maximum forces are not achieved during grinding. A magnitude of load $100 \mathrm{~N}$ was selected considering these reasons and simulating other in-vitro studies $(28,30)$.

The results of our study stated that, on comparing between standard implant length and short implants, according to BioHPP restorations, when vertical load was applied on the central fossa, it was found that, the difference in the microstrains developed around the implants was not statistically significant. Adversely, there was significant increase in microstrains values for short implants than standard implant length with zirconia restorations.

As for the BioHPP restorative material, when mesial load was applied, no significant difference was found between the microstrains developed around standard implant length and short implants. While for zirconia restorations, the difference between the microstrains developed around the two groups was statistically significant.

Moreover, when distal load was applied, there was no statistical significant difference in the microstrains values between standard implant length and short implants, according to BioHPP. While, the difference in microstrains was found to be statistically significant for zirconia restoration.

The statistically significant differences in microstrains values on comparing between short and standard implant length, only occurred with zirconia restorations. This was explained by Soliman TA et al. (31), who reported that, crown materials with high modulus of elasticity (as Zirconia) transfer high values of the applied load to underlying bone. Conversely, BioHPP act as a shock absorber, it reduces the stress caused by natural forces. The elastic modulus of BioHPP lies in the range of $4000 \mathrm{MPa}$, which very strongly resembles the elasticity of human bone, so that the chewing forces are, therefore cushioned (32). It has lower modulus of elasticity than zirconia (28). Hence, it reduces the stresses generated on the bone, absorbs more energy from the applied load, and transfers less energy to the bone.

Considering oblique load that was applied at $45^{\circ}$ on the central fossa, this study stated that, upon comparing between standard implant length and short implants, the difference was found to be statistically not significant for both BioHPP and zirconia.

Furthermore, when the average of all kind of loads was analyzed, it was observed that, the microstrains developed around short implants were higher than those occurred around standard implant length for both BioHPP and zirconia, however, the difference was statistically not significant.

According to the results of this study, we came to a conclusion that, the performance of short implants is comparable to regular implants, and that, short implants may be successfully used to support a single crown in the posterior region of the maxilla. Short dental implants can be used as an alternative treatment option in sites where long implants could not be placed because of limited available bone, reducing the need for complex surgical adjunctive procedures.

From our point of view, short implants offer several surgical advantages over longer implants. The use of short implants in the posterior jaw region reduces the need for bone augmentation procedures prior to implant placement in the maxilla and the mandible. Shorter implants reduce the surgical risk of surgical complications. The decreased length of the drills and implants means that, the osteotomy preparation carries less risk of overheating the bone, and makes easier the insertion of drills and implants in small intra arch spaces. In case of apical root proximity, short implants can be the only possible choice. All these factors make short implants a highly recommended restorative option.

The results of this study agreed with the systemic review (33) which stated that, short dental implants $(6 \mathrm{~mm}$ to $8 \mathrm{~mm}$ ) can be used successfully to support single or multiple fixed reconstructions or overdentures in atrophic maxillae and mandibles. The use of short dental implants reduces the need for advanced and complicated procedures, which reduces complications, costs, treatment time, and morbidity.

Recently, Lai et al. (34), found that, the 6 and $8 \mathrm{~mm}$ length implants showed, respectively, a cumulative survival rate of 97 and $98.5 \%$, with no differences in regard to the implanted jaw. In addition, Gulje et al. (35), examined 41 patients randomly allocated to receive an $11 \mathrm{~mm}$ implant in combination with maxillary sinus floor elevation surgery or to receive a $6 \mathrm{~mm}$ implant without any grafting in the posterior maxilla. At the 12th month evaluation implant, survival rate was $100 \%$ in both groups.

Hagi and Telleman et al. $(36,37)$, in their recent studies concluded that, dental implant surface geometry is a major determinant of how well a short implant performs. Implant diameter should also be considered as an important clinical variable. It has been suggested that, increased implant diameter could compensate for reduced length.

On the contrary, a finite element analysis reported higher stress transfer with short implants than with the standard implants. They observed that, stress distribution was wider, and covered more area of the cortical bone with the standard implants than with the short implants (38). 
In addition, this study observed that, on comparing between the mean values of microstrains of the average of central and oblique loads in standard implant length, according to BioHPP and zirconia restorations, the magnitude of stresses under oblique loading was found to be higher than that under axial loading, and the difference was statistically significant for both materials.

On the contrary, the same comparison was made for short implants, according to BioHPP, and the difference was found to be statistically not significant. However, the magnitude of stresses under oblique load was much higher than that under axial load for zirconia restoration, and the difference was found to be statistically significant.

This study concluded that, the component of oblique load is important to be considered when load is applied to dental implants, and should be kept to a minimum while designing restorations as these forces were seen to be the ones which could be detrimental to the maintenance of bone around implants. Application of biomechanical principles such as; reducing the cantilever length, passive fitting of prostheses, narrowing the buccolingual/ mesiodistal dimensions of the prosthesis, reducing cusp inclination, and centering occlusal contacts, can reduce occlusal overload and prevent complications (39).

The results of this study also showed that, on comparing between BioHPP and zirconia for standard implant length, it was found that, the difference was statistically not significant. Similarily, the difference was insignificant for short implants. These results occurred when the load was applied axially on the central fossa, mesial marginal ridge, and distal marginal ridge, and obliquely at $45^{\circ}$ as well as the average of all kind of loads.

This agrees with Bankoglu Güngör and Yılmaz (40), who reported that, prosthetic materials do not change the stress distributions on bones and that, implant survival is not affected by the prosthetic material. However, zirconia restorations transfer more stresses to short implants, when being compared with standard implant length, than does BioHPP. Therefore, it is recommended to use BioHPP prosthesis, instead of zirconia for short implants as BioHPP act as a shock absorber, while zirconia has high modulus of elasticity.

\section{CONCLUSION}

1. Short implants represent successful treatment modality for single tooth restoration.

2. Oblique loads transfer more stresses to the supporting structure than axial loads.

3. According to the average of loads, there is no significant difference between BioHPP and zirconia on the strains developed around short and standard implant length.

4. It is advisable not to use zirconia restorations with short implants because zirconia has higher modulus of elasticity and transfers more stresses to short implants than does BioHPP.

\section{CONFLICT OF INTEREST}

The authors declare that they have no conflicts of interest.

\section{REFERENCES}

1- de Carvalho EB, Herbst PE, Faria AC, Ribeiro RF, Costa PP, Tiossi R. Strain transfer behavior of different planning options for mandibular single-molar replacement. J Prosthet Dent. 2018 Feb;119(2):250-256.

2- Friberg B, Ivanoff CJ, Lekholm U. Inferior Alveolar Nerve Transposition in Combination with Brånemark replant Treatment. Int J Periodontics Restorative Dent. 1992 Dec 1;12(6):440-9.

3- Pozzi A, Sannino G, Barlattani A. Minimally invasive treatment of the atrophic posterior maxilla: a proof-ofconcept prospective study with a follow-up of between 36 and 54 months. J Prosthet Dent. 2012 Nov 30;108:286-97.

4- Block MS, Haggerty CJ. Interpositional osteotomy for posterior mandible ridge augmentation. J Oral Maxillofac Surg. 2009 Nov 30;67:31-9.

5- Hämmerle CH, Jung RE, Feloutzis A. A systematic review of the survival of implants in bone sites augmented with barrier membranes (guided bone regeneration) in partially edentulous patients. J Clin Periodontol. 2002 Dec 1;29:226-31.

6- Barone A, Toti P, Menchini-Fabris GB, Felice P, Marchionni S, Covani U. Early volumetric changes after vertical augmentation of the atrophic posterior mandible with interpositional block graft versus onlay bone graft: A retrospective radiological study. J Craniomaxillofac Surg. 2017 Sep;45:1438-47.

7- Man Y, Wang T, Mo A, Qu Y. Implant over implant: An alternative method for solving malpositioned osseointegrated implants at the sinus floor. J Prosthet Dent. 2014 Oct 31;112(4):731-5.

8- Mezzomo LA, Miller R, Triches D, Alonso F, Shinkai RS. Meta-analysis of single crowns supported by short ( $<10 \mathrm{~mm}$ ) implants in the posterior region. J Clin Periodontol. 2014 Feb 1;41:191-213.

9- Lee SA, Lee CT, Fu MM, Elmisalati W, Chuang SK. Systematic review and meta-analysis of randomized controlled trials for the management of limited vertical height in the posterior region: short implants (5 to $8 \mathrm{~mm}$ ) vs longer implants $(>8 \mathrm{~mm})$ in vertically augmented sites. Int J Oral Maxillofac Implants. 2014 Sep $1 ; 29 ; 1085-97$.

10-Lemos CA, Ferro-Alves ML, Okamoto R, Mendonça MR, Pellizzer EP. Short dental implants versus standard dental implants placed in the posterior jaws: A systematic review and meta-analysis. J Dent. 2016 Apr 30;47:8-17.

11-Morand M, Irinakis T. The challenge of implant therapy in the posterior maxilla: providing a rationale for the use of short implants. J Oral Implantol. 2007 Oct;33:257-66.

12-Schulte J, Flores AM, Weed M. Crown-to-implant ratios of single tooth implant-supported restorations. J Prosthet Dent. 2007 Jul 31;98(1):1-5.

13-Blanes RJ. To what extent does the crown-implant ratio affect the survival and complications of implantsupported reconstructions? A systematic review. Clin Oral Implants Res 2009;20:67-72.

14-Gonçalves TM, Bortolini S, Martinolli M, Alfenas BF, Peruzzo DC, Natali A et al. Long-term short implants performance: systematic review and meta-analysis of the essential assessment parameters. Braz Dent J. 2015 Aug;26:325-36. 
15-Gehrke SA. Importance of crown height ratios in dental implants on the fracture strength of different connection designs: an in vitro study. Clin Implant Dent Relat Res. 2015 Aug 1;17:790-7.

16-Gentile MA, Chuang SK, Dodson TB. Survival estimates and risk factors for failure with $6 \times 5.7-\mathrm{mm}$ implants. Int J Oral Maxillofac Implants. 2005;20: 9307.

17-Ali Al-Hashedi A, Bai Taiyeb Ali T, Yunus N. Short dental implants: an emerging concept in implant treatment. Quintessence Int. 2014 Jun 1;45:499-514.

18-Zoidis P, Papathanasiou I. Modified PEEK resin-bonded fixed dental prosthesis as an interim restoration after implant placement. J Prosthet Dent. 2016 Nov 30;116:637-41.

19-Heimer S, Schmidlin PR, Roos M, Stawarczyk B. Surface properties of polyetheretherketone after different laboratory and chairside polishing protocols. J Prosthet Dent. 2017 Mar 31;117:419-25.

20-Fabbri G, Sorrentino R, Brennan M, Cerutti A. A novel approach to implant screw-retained restorations: adhesive combination between zirconia frameworks and monolithic lithium disilicate. Int J Esthet Dent. 2014; 9:490-505.

21-Stafford GD, Glantz PO. Intraoral strain gauge measurements on complete dentures: a methodological study. J Dent.1991 Apr 1;19:80-4.

22-Di Stefano DA, Arosio P, Gastaldi G, Gherlone E. The insertion torque-depth curve integral as a measure of implant primary stability: An in vitro study on polyurethane foam blocks. J Prosthet Dent. 2017 Jul 8.

23-Almeida KP, Delgado-Ruiz R, Carneiro LG, Leiva AB, Calvo-Guirado JL, Gómez-Moreno G et al. Influence of Drilling Speed on Stability of Tapered Dental Implants: An Ex Vivo Experimental Study. Int J Oral Maxillofac Implants. 2016 Jul 1;31:795-8.

24-Sarendranath A, Khan R, Tovar N, Marin C, Yoo D, Redisch $\mathrm{J}$ et al. Effect of low speed drilling on osseointegration using simplified drilling procedures. $\mathrm{Br}$ J Oral Maxillofac Surg. 2015 Jul 1;53:550-6.

25-Nishioka RS, Vasconcellos LG, Jóias RP, Rode SD. Load-application devices: a comparative strain gauge analysis. Braz Dent J. 2015 Jun;26:258-62.

26-Huang HL, Chang YY, Lin DJ, Li YF, Chen KT, Hsu JT. Initial stability and bone strain evaluation of the immediately loaded dental implant: an in vitro model study. Clin Oral Implants Res. 2011 Jul 1;22:691-8.

27-Kola MZ, Shah AH, Khalil HS, Rabah AM, Harby NM, Sabra SA et al. Surgical templates for dental implant positioning; current knowledge and clinical perspectives Niger J Surg. 2015 Jan;21:1-5.

28-Kaleli N, Sarac D, Külünk S, Öztürk Ö. Effect of different restorative crown and customized abutment materials on stress distribution in single implants and peripheral bone: A three-dimensional finite element analysis study. J Prosthet Dent. 2018 Mar 1;119:437-45.
29-El-Anwar MI, El-Mofty MS, Awad AH, El-Sheikh SA, El-Zawahry MM. The effect of using different crown and implant materials on bone stress distribution: a finite element study. Egyptian Journal of Oral and Maxillofacial Surgery. 2014 May 1;5:58-64.

30-Barão VA, Delben JA, Lima J, Cabral T, Assunção WG. Comparison of different designs of implant-retained overdentures and fixed full-arch implant-supported prosthesis on stress distribution in edentulous mandiblea computed tomography-based three-dimensional finite element analysis. J Biomech. 2013 Apr 26;46:1312-20.

31-Soliman TA, Tamam RA, Yousief SA, El-Anwar MI. Assessment of stress distribution around implant fixture with three different crown materials. Tanta Dental Journal. 2015 Dec 1;12:1-10.

32-Bechir ES, Bechir AN, Gioga CH, Manu R, Burcea AL, Dascalu IT. The advantages of BioHPP polymer as superstructure material in oral implantology. Materiale Plastice. 2016 Sep 1;53:394-8.

33-Edher F, Nguyen CT. Short dental implants: A scoping review of the literature for patients with head and neck cancer. J Prosthet Dent. 2018 May;119:736-42.

34-Lai HC, Si MS, Zhuang LF, Shen H, Liu YL, Wismeijer D. Long-term outcomes of short dental implants supporting single crowns in posterior region: a clinical retrospective study of 5-10 years. Clin Oral Implants Res. 2013 Feb; 24:230-7.

35-Guljé FL, Raghoebar GM, Vissink A, Meijer HJ. Single crowns in the resorbed posterior maxilla supported by either 6-mm implants or by 11-mm implants combined with sinus floor elevation surgery: a 1-year randomised controlled trial. Eur J Oral Implantol. 2014 Sep 1;7:24755.

36-Hagi D, Deporter DA, Pilliar RM, Arenovich T. A targeted review of study outcomes with short $(\leq 7 \mathrm{~mm})$ endosseous dental implants placed in partially edentulous patients. J Periodontol. 2004 Jun 1;75:798804.

37-Telleman G, Raghoebar GM, Vissink A, Den Hartog L, Huddleston Slater JJ, Meijer HJ. A systematic review of the prognosis of short $(<10 \mathrm{~mm})$ dental implants placed in the partially edentulous patient. J Clin Periodontol. 2011 Jul 1;38:667-76.

38-Bourauel C, Aitlahrach M, Heinemann F, Hasan I. Biomechanical finite element analysis of small diameter and short dental implants: extensive study of commercial implants. Biomedizinische Technik/Biomedical Engineering. 2012 Feb 1;57:21-32.

39-Paliwal S, Saxena D, Mittal R, Chaudhary S. Occlusal Principles and Considerations for Implants: An Overview. Journal of Academy of Dental Education. 2014 Dec 1; 1:17-21.

40-Güngör MB, Y1lmaz H. Evaluation of stress distributions occurring on zirconia and titanium implant-supported prostheses: a three-dimensional finite 
element analysis. J Prosthet Dent. 2016 Sep 1;116:346-

55.

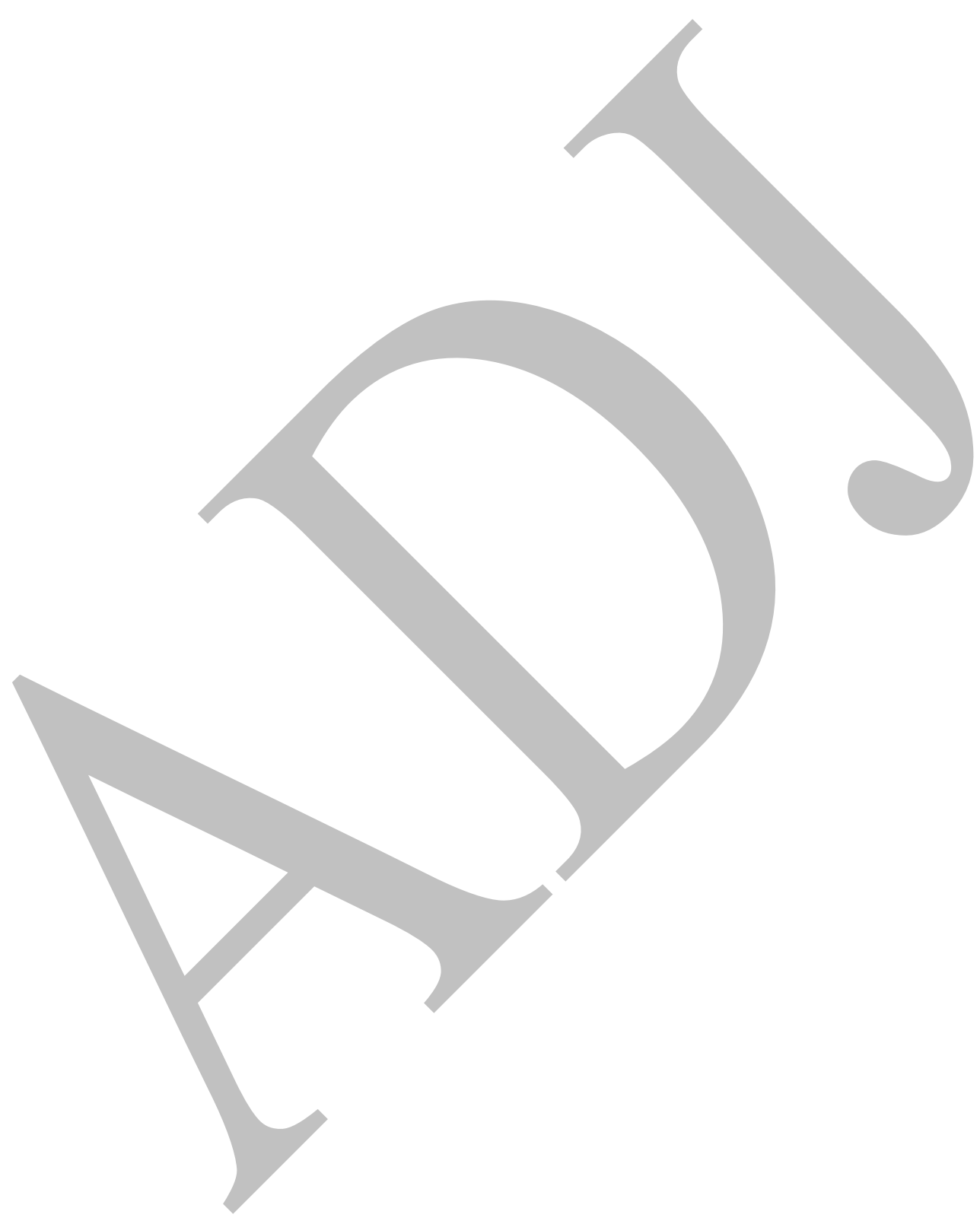

